DOI: https://doi.org/10.46630/phm.12.2020.21

Ana M. Vujović ${ }^{1}$

Article de recherche

Université de Belgrade

УДК 811.133.1'276.6:371.3

Faculté de formation des maîtres ${ }^{2}$

811.133.1'243(497.11)

Reçu : le 22/1/2020

\title{
LA PERSPECTIVE ACTIONNELLE DANS L'ENSEIGNEMENT/APPRENTISSAGE DU FRANÇAIS DE SPÉCIALITÉ
}

L'approche actionnelle s'est imposée comme l'approche didactique la plus conforme à la construction de la cognition, puisqu'elle met en coordination les activités physiques, mentales et langagières. Pour la réalisation d'une tâche qui pose un problème, les apprenants sont obligés de mettre en œuvre des connaissances, des savoirs et des compétences qu'ils n'avaient pas au début et qui se construisent durant la résolution de ce problème. Cette contribution se propose pour objectif de situer la perspective actionnelle et l'approche par les tâches tant du point de vue de l'actualité des réalisations que des perspectives pour la classe du français de spécialité ou du français sur objectif spécifique. Nous analysons d'abord les notions-clés et utilisons ensuite la méthode descriptive et analytique en présentant quelques exemples de tâches possibles dans la formation de futurs professeurs des écoles (serbes et français). Notre hypothèse est que les démarches de recherche (en ligne ou ailleurs) favoriseraient un meilleur apprentissage dans le domaine de langue de spécialité. Les résultats de notre travail montrent que l'exécution des tâches liées à leur future profession motive les étudiants - futurs professeurs des écoles qui apprennent le français en tant que langue de spécialité. Les étudiants prennent conscience du fait que, en même temps qu'ils sont mobilisés sur le résultat linguistique, ils sont en train d'apprendre des choses nouvelles dans le domaine de leur future profession.

Mots-clés : perspective actionnelle, tâche, compétence, français de spécialité, étudiants, professeurs des écoles

\section{Définitions des notions-clés}

Dans le monde contemporain, surtout dans un cadre professionnel, il ne s'agit plus d'apprendre une langue pour la parler plus tard, il s'agit de la parler

\footnotetext{
${ }^{1}$ ana.vujovic@uf.bg.ac.rs

${ }^{2}$ Cette contribution fait partie des recherches menées dans le cadre du projet Concepts et stratégies de l'assurance de la qualité de l'éducation de base, numéro179020D, financé par le Ministère de l'éducation, de la science et du développement technologique de la République de Serbie.
} 
afin de l'apprendre. Les nouvelles situations sociales des apprenants/usagers sont celles de vivre ensemble (dans une société multilingue et multiculturelle) et de travailler ensemble (dans l'espace universitaire et professionnel en voie d'intégration). Donc, l'objectif de la didactique des langues devient plus général : mobiliser les capacités créatrices des enseignants et des apprenants et rendre l'enseignement/apprentissage plus efficace. Les documents sont au service des activités et non le contraire, on parle davantage des compétences et non seulement des connaissances des apprenants. Les savoirs s'acquièrent au travers des tâches et la co-action prend son sens dans une démarche de projet qui permet d'aboutir à des réalisations concrètes et à la conquête de l'autonomie langagière. Étant occupés par le « comment faire » pour achever le travail, les apprenants se soucient moins du « comment dire »; ils mobilisent leurs compétences verbales mais utilisent aussi la communication non-verbale. (VUJOVIĆ 2015 : 295)

Au sein du FLE on distingue le français général (FG) et le français sur objectif spécifique (FOS) qui a un objet précis centré sur certaines situations et compétences cibles ; les contenus sont souvent nouveaux pour l'enseignant qui est obligé de prendre contact avec les acteurs du milieu étudié et d'élaborer le matériel pédagogique. Le FOS n'est pas un domaine séparé du FLE mais un sous-champ du FLE. « Le meilleur moyen de comprendre ce qu'est la méthodologie communicative consiste, sans doute, à se pencher sur la mise en place d'un programme de FOS. » (MANGIANTE, PARPETTE 2004 : 159)

Il faudrait d'abord distinguer les notions très proches mais distinctes qui sont :

a) Français sur objectif spécifique (FOS) qui est lié à une demande / un besoin clairement identifié(e), besoin extérieur à l'apprentissage de la langue ;

b) Français de spécialité (FS) qui est lié à un domaine professionnel mais sans besoin clairement identifié (concernant les situations de communication ou les compétences). (VUJOVIĆ 2019 : 150)

Les deux concernent un type d'enseignement axé sur le lexique spécialisé, la grammaire de spécialité, la maîtrise des actes de parole de la pratique des genres discursifs du domaine. On considère de plus en plus souvent qu'il est possible de les enseigner à tous les niveaux d'apprentissage de la langue, même aux débutants. Dans l'enseignement-apprentissage du FOS et du FS le choix des activités dépend du domaine professionnel concret (vu que la compréhension et/ou la production écrite peuvent avoir un rôle très important). Les étudiants des facultés non-philologiques peuvent se trouver dans les deux situations, c'est pourquoi leurs enseignants du français (ainsi que d'autres langues étrangères) cherchent tout le temps de nouvelles voies pour les aider à maîtriser les compétences linguistiques nécessaires pour leur future profession. Ces voies diffèrent selon les profils professionnels, mais aussi selon chaque groupe d'étudiants, ce qui représente la difficulté principale pour les enseignants qui 
n'arrêtent pas de s'adapter aux besoins et aux situations différents. Ils sont donc obligés, avant tout, d'analyser les besoins concrets de leurs étudiants, prendre en considération l'arrière-plan culturel des discours échangés dans les situations communicatives cibles qui peuvent être très différentes (dans une école élémentaire française ou francophone, dans une INSPÉ ${ }^{3}$, dans un entourage francophone de la vie quotidienne et universitaire, etc.), mais sans oublier que tous les étudiants n'auront pas besoin de communiquer directement avec leurs homologues francophones et ne devront qu'utiliser les documents écrits et oraux disponibles en ligne ou ailleurs. Ensuite l'enseignant procède à la collecte des données qui est l'étape la plus spécifique à l'élaboration d'un programme du FOS/FS. Cette étape confirme, complète ou modifie largement l'analyse des besoins faite par l'enseignant-concepteur du programme, qui doit sortir de son cadre habituel et bien connu pour entrer en contact avec un milieu mal connu, voire inconnu, pour y obtenir des informations dont il a besoin. La collecte des données sur le terrain n'est pas facile et suppose souvent aussi un travail de reportage, de recherche d'explications auprès des acteurs des milieux concernés. On distingue ainsi les discours existants (à enregistrer, comme les cours magistraux, ou à recueillir directement, comme les prises de notes des étudiants ou les devoirs) et les discours sollicités (interviews, témoignages) nécessaires lorsque le concepteur n'a pas accès aux premiers (MANGIANTE, PARPETTE 2004 ; VUJOVIĆ 2011). Heureusement, la coopération de notre faculté (Faculté de formation des maîtres de l'Université de Belgrade) avec plusieurs établissements universitaires en France nous facilite ce travail et nous permet d'extraire les données essentielles.

Il reste enfin à l'enseignant/élaborateur du programme d'analyser toutes ces données, d'en choisir celles qui conviennent le mieux aux besoins concrets et de concevoir des activités didactiques. L'élaboration didactique, quant à elle, repose sur les principes méthodologiques suivants: les activités sont au service de l'analyse des besoins elle-même réalisée en fonction des situations de communication identifiées ; l'élaboration didactique s'effectue en lien étroit avec les informations issues de l'analyse des données collectées ; elle procède de la méthodologie communicative (documents authentiques, communication naturelle dans le groupe) et elle suppose une participation maximale des apprenants et le retrait de l'enseignant. (MANGIANTE, PARPETTE 2004).

$\mathrm{Vu}$ toutes ces spécificités et difficultés de l'enseignement/apprentissage du FOS/FS, il nous parait que la perspective actionnelle et l'approche par les tâches s'y prêtent le mieux.

La notion de la tâchen'est pas nouvelle en didactique des languesétrangères. C'est une activité authentique que l'apprenant réalise individuellement, en

${ }^{3}$ INSPÉ - Institut National Supérieur du Professorat et de l'Éducation, institution universitaire pour la formation de futurs enseignants. 
binôme ou en groupe et qui, ce qui est essentiel, fait sens pour lui. Le résultat concret de la réalisation de cette tâche permet de voir dans quelle mesure il y a eu apprentissage et quels étaient les points faibles en méthodologie. M.S. Russo dit que d'après certains auteurs (R. Ellis, F. Demazière, J.-P. Narcy Combes), il est possible de construire des macro-tâches s'inspirant de plusieurs situations différentes, que l'on peut repérer dans la vie quotidienne ou professionnelle, ainsi que les micro-tâches qui sont plus circonscrites et moins réalistes. « Elles incluent gestion du sens et focalisation de l'attention sur des phénomènes importants. Elles sont analytiques, mais ne peuvent pas être déconnectées de toute mise en œuvre dans une situation d'énonciation, si l'on vise aux déclenchements des processus activant l'acquisition langagière. Par conséquent, l'apprenant sera invité à repérer, à percevoir dans quelle mesure son observation le sert et le dessert. Cela implique des avantages lors des échanges langagiers hors tâches. Le but des micro-tâches est de permettre l'accomplissement du travail à exécuter pendant les phases de pré/post-tâche. Le rôle de l'enseignant concernera donc le guidage, la didactisation, la pédagogisation du contenu, afin d'aider l'apprenant à accélérer le processus d'apprentissage. » (RUSSO 2013)

Dans l'apprentissage de la langue on insiste surtout sur l'accomplissement des tâches qui vont permettre le développement des compétences, sur l'utilisation des moyens linguistiques qu'on ne maîtrise pas encore afin de se rendre capable de les utiliser. La seule expérience pratique ne suffit pas ; l'apprenant a besoin d'un travail, d'une élaboration où intervient l'intelligence discursive, le langage qui permet l'activité de connaissance. Il est important que ces tâches mobilisent l'intérêt des étudiants et déclenchent des opérations mentales génératrices d'apprentissages, c'est pourquoi il faut que la tâche soit une activité contextualisée qui présente une situation de la vie réelle, un problème à résoudre et qui met en relation des interlocuteurs ayant des choses à se dire et des actions à produire ensemble. Il ne faut surtout pas oublier le rôle fondamental de la motivation dans l'apprentissage par tâches, vu que la tâche devrait provoquer la curiosité, susciter l'engagement pour atteindre un but.

C'est une réalisation concrète de type social qui permet à l'enseignant d'atteindre les objectifs linguistiques, culturels et pragmatiques proposés, une activité complexe qui oblige les étudiants à mobiliser des savoirs différents, à s'organiser, à chercher des réponses qui peuvent être diverses et imprévisibles. Pour la réalisation de cette tâche les apprenants sont obligés de mettre en œuvre des connaissances, des savoirs et des compétences qu'ils n'avaient pas au début et qui se construisent dans la résolution de ce problème. Les apprenants doivent prendre conscience du fait que, en même temps qu'ils sont mobilisés sur le résultat, ils sont en train d'apprendre des choses nouvelles (c'est ce que Pierre Bange appelle la bifocalisation). (VUJOVIĆ 2015 : 297)

La perspective actionnelle et l'approche par les tâches s'effectuent en classe, mais en articulation avec les tâches effectuées en dehors de la classe. 
Ces tâches effectuées en classe présentent les caractéristiques globales suivantes : elles sont généralement choisies par l'enseignant en fonction de ses objectifs ; elles sont effectuées par un seul apprenant ou par plusieurs ; leur accomplissement donne lieu à une évaluation qui porte sur le fond (la tâche menée à bien avec succès), mais éventuellement également sur la forme (une réalisation linguistique correcte). Dans le CECR les tâches sont classées en trois catégories :

- les tâches de pré-communication pédagogique constituées d'exercices spécifiquement axés sur la manipulation décontextualisée des formes ;

- les tâches « pédagogiques communicatives » que l'on connaît bien depuis l'approche communicative et dans lesquelles les apprenants s'engagent dans un « faire-semblant accepté volontairement » pour jouer le jeu de l'utilisation de la langue-cible, se préparant ainsi à mener à bien

- les tâches « proches de la vie réelle » choisies en fonction des besoins de l'apprenant hors de la classe ou du contexte d'apprentissage (par exemple, lire un mode d'emploi ou prendre des notes pendant un cours magistral).

" Les tâches pédagogiques communicatives (contrairement aux exercices formels hors contexte) visent à impliquer l'apprenant dans une communication réelle, ont un sens (pour l'apprenant), sont pertinentes (ici et maintenant dans la situation formelle d'apprentissage), exigeantes mais faisables (avec un réajustement de l'activité si nécessaire) et ont un résultat identifiable (ainsi que d'autres, moins évidents dans l'immédiat). Les activités de ce type peuvent avoir pour complément des tâches intermédiaires « métacommunicatives » telles que les échanges autour de la mise en œuvre de la tâche et la langue utilisée pour la mener à bien. Cela suppose que l'apprenant contribue à la sélection, à la gestion et à l'évaluation de l'activité ce qui, dans la situation d'apprentissage d'une langue, peut devenir partie intégrante des activités elles-mêmes. » (CECR : 121)

2. Quelques exemples de tâches dans l'enseignement/apprentissage du français de spécialité

Ces dernières années l'internationalisation de l'enseignement supérieur et l'expansion de la mobilité étudiante influencent fortement le fonctionnement des universités européennes. Grâce aux programmes de coopération développée entre la Faculté de formation des maîtres de l'Université de Belgrade et les INSPÉ de Bordeaux et de Lyon, nos étudiants peuvent intégrer 
les programmes de mobilité universitaire Erasmus+ (au niveau de Master 1 et 2), ainsi qu'exercer une partie de leur stage professionnel dans un milieu francophone. En même temps nous recevons les stagiaires français, étudiants au Master 1 et 2 de l'INSPÉ de Lyon, qui viennent faire un stage pratique accompagné à notre faculté et dans nos écoles maternelles et élémentaires.

Dans le processus de l'organisation de ces coopérations, notre premier objectif a été de définir les besoins de tous ces étudiants, serbes et français. Évidemment, les étudiants serbes sont seuls à devoir développer les compétences linguistiques et nous avons pensé que justement dans ce domaine leur contact avec les locuteurs natifs du français serait précieux. Ce passage d'un apprentissage individuel à un apprentissage collaboratif et solidaire (de la centration sur l'apprenant à la centration sur le groupe) trouve son expression dans l'approche par tâches, mais n'est jamais facile et simple à organiser. En plus, les étudiants serbes et français se trouvant éloignés sauf au moment de leur rencontre à Belgrade ou en France, l'enseignant à l'intérêt à demander de l'aide aux Français venant en stage à Belgrade.

À part les informations qu'on peut trouver sur les sites des Académies, des universités et des INSPÉ, ainsi que de l'Institut français et de l'AUF (programmes d'études ; documents et formulaires différents : Candidature pour les étudiants entrants, Contrat de mobilité, Visa de long séjour, Fiche de situation familiale, Fiche d'information, Appel à candidatures pour les bourses de mobilité d'études, assurance maladie, réclamations ; règlements des universités et des résidences universitaires ; vidéos des cours magistraux et des travaux dirigés ; brochures ou sites internet des bibliothèques etc.), il y a des documents impossibles à trouver en ligne (tels travaux écrits des étudiants, manuels utilisés dans l'enseignement à l'INSPÉ, fiches d'évaluation et de notation, exemples annales des examens des sessions précédentes ; exemples de $\mathrm{CV}$ et de lettres de motivation comme modèle de rédaction etc.). Alors on demande aux étudiants français de nous les envoyer ou apporter quand ils viennent en stage à Belgrade. Nous utilisons aussi des témoignages des étudiants serbes rentrant de France ou des stagiaires français venant en Serbie ; photos et vidéos des activités sportives, culturelles et artistiques, ainsi que plusieurs séquences du site Étudier en francophonie. On propose à nos étudiants voulant partir en mobilité pour intégrer le système universitaire français des contenus concernant : l'organisation des INSPÉ ; les documents et démarches nécessaires à la candidature pour une mobilité ou pour une bourse ; renseignements sur l'inscription, l'arrivée au campus, le logement, la restauration, le fonctionnement des CDI ; compréhension des discours universitaires (cours magistraux, travaux dirigés) ; informations essentielles sur les structures possibles des travaux écrits à l'université, l'évaluation, l'organisation des examens et le comportement par rapport aux professeurs/ 
étudiants/employés de différents services ; résolution des problèmes ; vie étudiante et activités extrascolaires (dans le campus ou en ville).

Pour l'autre groupe de nos étudiants, ceux qui partent faire un stage pratique, il est important plutôt de connaître l'organisation du système éducatif en France, surtout le fonctionnement des écoles maternelles et élémentaires où ils feront leur stage. C'est dans ce domaine que les témoignages des stagiaires français sont précieux, surtout de ceux qui sont au Master 2 et qui exercent déjà leur métier de professeur des écoles. Tous ces étudiants ont déjà une expérience supérieure à celle de l'enseignant du FOS/FS dans le champ professionnel traité et sont en mesure d'alimenter une partie des activités par leurs propres informations. Comme le temps prévu pour la formation est souvent très court, il est essentiel de développer des pratiques de classe les plus rentables en termes d'apprentissage.

De la pratique de l'oral dialogué (situation d'accueil à l'université ou dans une école, par exemple), à la compétence de lecture (des programmes, des documents administratifs, des manuels etc.), en passant par une compétence combinant la compréhension orale, la lecture, voire la production écrite (pour les étudiants en mobilité universitaire), la diversité des tâches et des activités à proposer est grande. L'approche communicative a pour principe d'introduire le plus possible dans la classe un fonctionnement d'échanges existant en situation naturelle. Et l'approche par tâches permet que ces échanges soient vraiment proches de la vie réelle.

Donc ce qu'on propose aux étudiants serbes comprend d'abord les tâches de pré-communication pédagogique constituées d'exercices lexicaux spécifiquement axés sur l'éducation en France, la formation et le métier des professeurs des écoles, le vocabulaire de la vie scolaire et universitaire. On passe ensuite aux tâches pédagogiques communicatives (jeux de rôles, dialogues inventés par les étudiants ou tirés de sites différents) dans lesquelles les étudiants jouent le jeu de l'utilisation de la langue française et se préparent ainsi à mener à bien les tâches encore plus proches de la vie réelle qu'ils feront en groupe et ensemble avec les stagiaires français. Ceci exige plus d'échanges entre étudiants et une organisation différente du travail (avec une distribution de deux ou plusieurs documents au lieu d'un seul de manière à amener les apprenants à échanger en sous-groupe les informations recueillies et à réaliser une tâche ensemble).

Sans prétendre à l'exhaustivité, nous présentons ici quelques exemples de tâches effectuées avec le public choisi pour notre travail de terrain - les étudiants serbes et français. Il s'agit de proposer des activités proches de leurs centres d'intérêt et portant sur les exigences de la vie de tous les jours, susceptibles de motiver les étudiants.

1. 1. Tâche $1:$ On prend plusieurs documents administratifs nécessaires aux étudiants serbes voulant partir en France (Candidature pour les 
étudiants entrants, Contrat de mobilité, Visa de long séjour, Fiche de situation familiale, Fiche d'information, Appel à candidatures pour les bourses de mobilité d'études...) ; on explique le lexique inconnu et demande de remplir les documents/formulaires présentés. Si nécessaire, on demande de l'aide aux étudiants français ou à nos étudiants qui ont déjà été en mobilité universitaire en France.

2. Tâche 2 : Les étudiants français montrent des exemples de plusieurs formes des $\mathrm{CV}$ et des lettres de motivation en français et expliquent ce qui est considéré être le plus important. Ensuite les étudiants serbes écrivent leur CV et leur lettre de motivation; suit le travail d'analyse et de contrôle.

3. Tâche 3 : Préparation des diaporamas sur la formation des professeurs des écoles en Serbie et en France. Les étudiants français présentent l'organisation des INSPÉ et leur programme MEEF (Métiers de l'enseignement, de l'éducation et de la formation); les étudiants serbes présentent leur formation à la faculté. On discute des différences et des ressemblances, surtout en ce qui concerne le stage pratique.

4. Tâche 4 : Trouver sur le site internet des bibliothèques (universitaires et autres) des informations et des publications au sujet d'un travail écrit proposé par le professeur.

5. Tâche 5: Préparation des diaporamas présentant les systèmes scolaires en France et en Serbie (organisation du travail dans des écoles maternelles et élémentaires); on distingue les avantages et les désavantages des deux systèmes. Cette tâche est destinée surtout aux étudiants serbes partant en stage à Lyon et aux étudiants français venant en stage à Belgrade.

6. Tâche 6 : Analyse des examens des sessions précédentes, présentation des fiches d'évaluation et de notation, tout dans le but d'une meilleure préparation des étudiants serbes partant en mobilité.

7. Tâche 7 : Analyse d'un mémoire français (structure, éléments obligatoires) et comparaison avec nos exigences ; travail de synthèse de toutes les caractéristiques essentielles.

8. Tâche 8 : Prendre les photos ou faire des vidéos des activités sportives, culturelles et artistiques des étudiants des deux pays, avec les explications de ces activités.

Le but de ces tâches est avant tout de déclencher un processus permettant aux étudiants de s'approprier le vocabulaire utilisé dans les professions liées à l'éducation et à l'enseignement, ainsi que d'apprendre à rechercher des informations précises à ce sujet en langue française (compréhension écrite sélective). Cette recherche a été effectuée en janvier 2019 avec les étudiants de 
deuxième année de la Faculté de formation des maîtres (niveau A2+ et B1 selon le CECR). Nous avons divisé 29 étudiants en deux groupes : le premier groupe (15 étudiants) avait pour tâche de répondre à certaines questions après avoir cherché et consulté les documents en ligne concernant leur future profession, tandis que le deuxième groupe (14 étudiants) n'avait pas cette expérience. Notre hypothèse était que les démarches de recherche en ligne favoriseraient l'apprentissage des mots concernant les informations repérées. Cette hypothèse a été confirmée surtout par une meilleure compréhension des supports, mais aussi par des productions langagières des étudiants (connaissance atteinte du vocabulaire; utilisation appropriée du vocabulaire, des tournures et des acronymes ; niveau grammatical et syntaxique; phrases plus correctes et parfois plus élaborées).

Les résultats de notre recherche indiquent qu'il est possible d'envisager l'organisation des séances au cours desquelles l'étudiant, s'exprimant en français, se livre à des tâches susceptibles de le motiver, lui permettant de connaître le discours et le milieu d'action propres aux futurs professionnels dans le domaine scientifique/professionnel. Dans le travail il faut toujours: utiliser des supports modernes, documents authentiques et actuels concernant des sujets potentiellement utiles dans la vie réelle, varier le contenu des séances pour motiver les étudiants.

Ne négligeons pas l'importance de l'enthousiasme et de l'investissement des étudiants, lors de l'exécution des tâches : afin que l'apprentissage ne soit pas lié uniquement aux résultats des productions réalisées, il est important d'établir des relations productives entre enseignant et apprenants et de travailler dans des conditions non anxiogènes, favorisant la concentration. Les résultats des tâches devraient être utilisés en tant qu'éléments contribuant à évaluer les étudiants, ou bien à choisir des micro-tâches d'entraînement ou de rattrapage. De cette façon, il serait possible de mettre en place des stratégies d'enseignement/apprentissage conformes à leurs exigences. Cette démarche nous semble adéquate aussi pour la planification du travail à proposer à des groupes hétérogènes d'étudiants fauxdébutants. Parmi les aspects positifs ayant concouru à la bonne réussite de cette recherche-action, nous pouvons indiquer le climat collaboratif instauré entre les étudiants, l'attitude constructive de nombreux apprenants, l'emploi des TIC et les sujets choisis. Cela a confirmé la validité de l'emploi des TIC et du rôle de l'enseignant en tant que facilitateur, concepteur, pourvoyeur et organisateur.

\section{Conclusion}

Les besoins d'expression en langues vivantes étrangères varient en fonction des métiers. Il devient donc indispensable de repérer les exigences contextuelles et d'adapter les services et les contenus aux destinataires des cours à planifier. Face au gain de terrain des formations linguistiques sur 
objectif universitaire, il serait possible de prendre en compte les besoins communicatifs des futurs professionnels même à partir du lycée (surtout du lycée professionnel), ce que propose M.S. Russo (RUSSO 2013).

Depuis des années on parle des politiques linguistiques des états et du plurilinguisme en danger, en essayant de mettre en place des mesures et des outils en faveur de l'apprentissage des langues étrangères, ainsi que des pratiques pédagogiques adaptées aux réalités du terrain. Ce qui inquiète les enseignants du FLE, c'est la baisse du nombre d'apprenants de la langue française dans tous les niveaux de l'éducation. Déjà en 2010, l'OIF dans son rapport affirme le recul du nombre d'apprenants de français en Europe. Le pessimisme et l'impuissance devant les actions et les mesures entreprises par les autorités éducationnelles et politiques sont évidents et ne font que grandir. Les conclusions du séminaire international „Evolutions et perspectives des études de français et en français dans la zone Balkans/Europe du sudest", qui a eu lieu à Athènes en mai 2010, mais aussi celles de nombreux colloques internationaux plus récents traitant la position du français dans le monde, montrent que l'avenir du français est avant tout dans les domaines des activités professionnelles. Paradoxalement, chez nous les langues sur objectifs spécifiques ou les langues de spécialité sont le plus souvent considérées comme une discipline générale et on ne comprend pas leur importance pour les compétences professionnelles des étudiants, d'où vient leur mauvaise position dans notre éducation supérieure.

Un autre paradoxe : dans une société qui ne respecte pas la profession des enseignants, cette profession devient de plus en plus exigeante, car les enseignants devraient apprendre à s'informer, à se former continuellement, à s'autoévaluer et à porter un regard réflexif sur leurs pratiques. Les enseignants de FLE/FOS/FS essaient de s'adapter et de répondre aux exigences de plus en plus spécifiques concernant le contexte, le public, les conditions de travail, les besoins. Ils sont sortis de la classe pour devenir des médiateurs, des guides censés encadrer les apprenants dans le processus d'apprentissage, mais aussi bien des concepteurs de programmes et du matériel pédagogique (surtout pour le FOS/FS).

On oublie souvent qu'écouter un enseignant n'a jamais un impact aussi fort que d'aller soi-même chercher une information, la trouver, la classer, la comparer avec d'autres et finalement en faire une synthèse. On apprend soimême mais toujours avec les autres ; c'est pourquoi l'alternance de phases individuelles et collectives dans le travail permet de vérifier, de rassurer, de prendre confiance et de mesurer ainsi le chemin parcouru. La notion de la motivation est depuis toujours associée au processus d'apprentissage. Elle renvoie au mouvement, se rapporte à plusieurs dimensions de l'action. On distingue la motivation intrinsèque, concernant les comportements qui amènent 
à des satisfactions personnelles sans attente de récompense (par exemple, un élève s'investit dans une matière plutôt qu'une autre) et la motivation extrinsèque qui se définit dans la récompense de l'action, que ce soit en terme économique (avoir une bonne note) ou social (reconnaissance à l'école et dans la famille). On parle souvent du manque de motivation (surtout chez les apprenants), on se demande comment motiver les apprenants, parfois on se décourage et s'interroge même sur le sens d'apprendre. Comme la motivation est la réponse à des besoins de réussite, d'auto-efficacité et de perspective sur sa propre action, c'est peut-être justement dans la perspective actionnelle et dans l'approche par les tâches qu'il faut la chercher.

Donc, l'enseignant du français de spécialité et/ou du français sur objectifs spécifiques devient le concepteur de situations qui doit mettre en place des pratiques de classe dans lesquelles l'activité de l'étudiant est au centre. « Les besoins langagiers des apprenants sont à la base des choix des contenus à enseigner ; il est de même important de fournir l'occasion de vivre, en classe de langue, des situations de communication se rapprochant de celles de la vie quotidienne. » (De Koninck et Huot, dans RUSSO 2013) Nous pensons surtout à l'approche active et aux projets qui devraient permettre aux apprenants de se concentrer sur les contenus dont ils auront vraiment besoin dans leur future vie professionnelle. Quelques caractéristiques particulières de la tâche sont notamment son lien avec la réalité, la concrétude de son résultat, son efficacité potentielle dans le processus d'apprentissage, la diversité. Et les étudiants doivent prendre leur partie de responsabilité et comprendre qu'ils doivent s'investir davantage, sans attendre que quelqu'un d'autre leur « remplisse la tête » par des quantités de savoir. Ils doivent agir pour pouvoir s'approprier le savoir, entrer dans la classe en étant conscients de leur besoin de s'engager dans une activité intellectuelle, se poser des questions, se tromper et revenir sur leurs pas, quand cela est nécessaire.

\section{Bibliographie}

Cadre européen commun de référence pour les langues : apprendre, enseigner, évaluer. Conseil de l'Europe, Unité des Politiques linguistiques, Strasbourg, Les Éditions Didier, Paris, 2001. <https://rm.coe.int/16802fc3a8>

MANGIANTE, PARPETTE 2004 : MANGIANTE, Jean-Marc et Chantal PARPETTE. Le Français sur objectif spécifique : de l'analyse des besoins à l'élaboration d'un cours. Paris : Hachette, 2004.

PUREN 2004 : PUREN, Christian. " De l'approche par les tâches à la perspective co-actionnelle ». HARDY, Mireille (dir.) Les Cahiers de l'APLIUT. Actes du XXVe Congrès. Auch, 5-7 juin 2003, vol. XXIII, n ${ }^{\circ}$ $1,(2004): 10-26$. 
PUREN 2009 : PUREN, Christian. « Variations sur la perspective de l'agir social en didactique des langues-cultures étrangères ». Le français dans le monde - Recherches et applications, $\mathrm{n}^{\circ} 45$ (2009) : 154-167.

PUREN 2009 : PUREN, Christian. « Nouvelle perspective actionnelle et (nouvelles) technologies éducatives : quelles convergences... et quelles divergences ? ». Les langues modernes. <https://www.aplvlanguesmodernes.org/spip.php?article2673> 12.01.2020.

RUSSO 2013 : RUSSO, Maria Serafina. «Langue de spécialité, approche par tâches, contexte de travail : un enseignement/apprentissage propédeutique à l'insertion professionnelle ». Recherches en didactique des langues et des cultures. <http://journals.openedition.org/rdlc/1639> 12. 12. 2019.

VUJOVIĆ 2009 : VUJOVIĆ, Ana. « Le français en tant que langue de spécialité en Serbie ». Revue de philologie, Belgrade, 2009/2 numéro spécial, (2009) : 305-312.

VUJOVIĆ 2011 : VUJOVIĆ, Ana. « La collecte et l'analyse des données dans l'enseignement / apprentissage de FOS ». IGNJAČEVIĆ, Anđelka et al. (dir.) Jezik struke : Izazovi i perspektive, Beograd: Društvo za strane jezike i književnosti Srbije i Sekcija univerzitetskih nastavnika stranog jezika struke, (2011) : 133-141.

VUJOVIĆ 2015 : VUJOVIĆ, Ana. « De la tâche à l'activité intellectuelle consciente ». VINAVER-KOVIĆ, Milica et Veran STANOJEVIĆ (dir.) Les études françaises aujourd'hui (2014). Belgrade : Faculté de philologie de l’Université de Belgrade, (2015) : 295-305.

VUJOVIĆ 2018 : VUJOVIĆ, Ana. « Notions de pédagogie en français et en serbe ». Filolog IX 2018 18, Banja Luka, (2018) : 30-43.

VUJOVIĆ 2019: VUJOVIĆ, Ana. « Le Français sur objectifs universitaires pour les professeurs des écoles ». STANOJEVIĆ, Veran et Milica VINAVER-KOVIĆ (dir.) Les études françaises aujourd'hui, Faculté de philologie de l'Université de Belgrade, Serbie, 9-10 novembre 2018, 148-157.

Ана М. Вујовић

\section{АКЦИОНИ ПРИСТУП НАСТАВИ И УЧЕњУ ФРАНЦУСКОГ ЈЕЗИКА СТРУКЕ}

Будући да комбинује физичке, менталне и језичке активности, акциони приступ показао се као најподеснији за развијање когнитивних способности. Да би остварили задатак који представља неки проблем, ученици/ студенти морају да употребе сва своја знања и компетенције, како оне пре- 
тходно стечене тако и оне које се развијају током самог процеса решавања проблема. Наш рад за циљ има да представи пример акционог приступа који одликује извршавање задатака у настави/учењу француског као страног језика наставничке струке. Најпре анализирамо неколико кључних појмова (као што су: француски као језик струке, акциони приступ, појам задатка, подела задатака), а потом, користећи дескриптивну и аналитичку методу, приказујемо неколико примера могућих задатака које користимо у настави француског језика струке будућих васпитача и учитеља. Наша полазна хипотеза заснива се на тврдњи да самостално трагање за информацијама (како на интернету тако и у другим расположивим изворима) позитивно утиче на овладавање језичким вештинама, како у разумевању прочитаних докумената тако и у самосталном усменом и писменом изражавању (употребом одговарајућег речника и акронима, коришћењем правилних граматичких и синтаксичких конструкција, грађењем језички исправнијих и сложенијих реченица). Резултати нашег истраживања показују да обављање задатака који су повезани са њиховом будућом професијом мотивише студенте, јер су они у највећем броју свесни чињенице да њихово решавање задатих лингвистичких проблема уједно представља за њих и стицање нових стручних знања и компетенција.

Кључне речи: акциона перспектива, задатак, компетенција, француски језик струке, студенти, учитељи и васпитачи 